\title{
EXISTENCE OF SOLUTIONS OF SEMILINEAR DIFFERENTIAL EQUATIONS WITH NONLOCAL CONDITIONS IN BANACH SPACES
}

\author{
K. BALACHANDRAN AND M. CHANDRASEKARAN
}

\begin{abstract}
The aim of this paper is to prove the existence and uniquencess of local, strong and global solutions of a nonlocal Cauchy problem for a differential equation. The method of analytic semigroups and the contraction mapping principle are used to establish the results.
\end{abstract}

\section{Introduction}

The problem of existence of solutions of evolution equation with nonlocal conditions ir. Banach space has been studied first by Byszewski [5]. In that paper he has establised the existence and uniqueness of mild, strong and classical solutions of the following nonlocal Cauchy problem:

$$
\begin{gathered}
\frac{d u(t)}{d t}+A u(t)=f(t, u(t)), \quad t \in\left(t_{0}, t_{0}+a\right] \\
u\left(t_{0}\right)+g\left(t_{1}, t_{2}, \ldots, t_{p}, u(\cdot)\right)=u_{0}
\end{gathered}
$$

where $-A$ is the infinitesimal generator of a $C_{0}$ semigroup $T(t), t \geq 0$, in a Banach space $X, 0 \leq t_{0}<t_{1}<\cdots<t_{p} \leq t_{0}+a, a>0, u_{0} \in X$ and $f:\left[t_{0}, t_{0}+a\right] \times X \rightarrow X$, $g:\left[t_{0}, t_{0}+a\right]^{p} \times X \rightarrow X$ are given functions. Subsequently he has investigated the same type of problem to a different class of evolution equations in Banach spaces [3-7]. Here the symbol $g\left(t_{1}, \ldots, t_{p}, u(\cdot)\right)$ is used in the sense that in the place of '.' we can substitute only elements of the set $\left\{t_{1}, \ldots, t_{p}\right\}$.

The purpose of this paper is to prove the existence and uniqueness of local, strong and global solutions for a semilinear differential equation with nonlocal conditions of the form:

$$
\begin{aligned}
& \frac{d u(t)}{d t}+A u(t)=f(u(t)), \quad t \in(0, b] \\
& u(0)+g\left(t_{1}, t_{2}, \ldots, t_{p}, u\left(t_{1}\right), \ldots, u\left(t_{p}\right)\right)=u_{0}
\end{aligned}
$$

where $0 \leq t_{0}<t_{1}<\cdots<t_{p} \leq b$. For example,

$$
g\left(t_{1}, t_{2}, \ldots, t_{p}, u\left(t_{1}\right), \ldots, u\left(t_{p}\right)\right)=c_{1} u\left(t_{1}\right)+\cdots+c_{p} u\left(t_{p}\right)
$$

Received April 9, 1997.

1991 Mathematics Subject Classification. 34G20, 47H15.

Key words and phrases. Existence of solution, semilinear equation, nonlocal conditions. 
where $c_{i}(i=1, \ldots, p)$ are constants. In this case, equation (4) allows the measurements at $t=0, t_{1}, \ldots, t_{p}$ rather than just at $t=0$.

Here we assume that $-A$ is the infinitesimal generator of a bounded analytic semigroup of linear operator $T(t), t \geq 0$, in a Banach space $X$. The operator $A^{\alpha}$ can be defined for $0 \leq \alpha<1$ and $A^{\alpha}$ is a closed linear invertible operator with domain $D\left(A^{\alpha}\right)$ dense in $X$. The closedness of $A^{\alpha}$ implies that $D\left(A^{\alpha}\right)$ endowed with the graph norm of $A^{\alpha}$, that is the norm $\|x\|=\|x\|+\left\|A^{\alpha} x\right\|$, is a Banach space. Sine $A^{\alpha}$ is invertible its graph norm $\|\cdot\|$ is equivalent to the norm $\|x\|_{\alpha}=\left\|A^{\alpha} x\right\|$. Thus, $D\left(A^{\alpha}\right)$ equipped with the norm $\|\cdot\|_{\alpha}$, is a Banach space which we denote by $X_{\alpha}$. From the definition it is clear that $0<\alpha<\beta$ implies $X_{\alpha} \supset X_{\beta}$ and that the imbedding of $X_{\beta}$ in $X_{\alpha}$ is continuous. Throughout the paper we shall use the symbol $J=[0, b]$. The nonlinear operators $f: X_{\alpha} \rightarrow X, g\left(t_{1}, \ldots, t_{p}, u\left(t_{1}\right), \ldots, u\left(t_{p}\right)\right): J^{p} \times X_{\alpha}^{p} \rightarrow Y$ are given functions.

The motivation for an abstract theory such as this comes from the following partial differential equation:

$$
\begin{array}{ll}
v_{t}(x, t)-v_{x x}(x, t)=\sigma(v(x, t))_{x}, & 0<x<1 \\
v(0, t)=v(1, t)=0 & t>0 \\
v(x, 0)=v(x, 1)+s(x) & 0<x<1
\end{array}
$$

It is not true in general that $\frac{\partial}{\partial x}=A^{1 / 2}$, however [13] there exist a bounded linear operator $B$ from $X$ into itself such that $A^{1 / 2} B=\frac{\partial}{\partial x}$. Letting $G=B \sigma$ we can fit the above equations into the abstract theory developed in this paper.

The abstract theory one can find in the books $[8,9,12]$ handles partial differential equations of the above forms, however the theory illustrated in these works does not distinguish between the problems of the form

$$
v_{t}(x, t)-v_{x x}(x, t)=v(x, t)|v(x, t)|^{\beta-1}
$$

with nonlocal conditions and the above equations but with right hand side $\frac{\partial}{\partial x}[v(x, t)$ $\left.|v(x, t)|^{\beta-1}\right]$

As in $[1-3,7,8,10,11]$ the nonlocal condition (4) can be applied in physics with better effect than the classical condition $u(0)=u_{0}$ since condition (4) is usually more precise for physical measurements than the classical condition.

\section{Preliminaries}

It is known that equations $(3)-(4)$ are related to the integral equation

$$
u(t)=T(t) u_{0}-T(t) g\left(t_{1}, \ldots, t_{p}, u\left(t_{1}\right), \ldots, u\left(t_{p}\right)\right)+\int_{0}^{t} T(t-s) f(u(s)) d s, \quad t \geq 0
$$

where $T(t)$ is the semigroup of operators generated by $-A$. The solution $u(t)$ of equation $(5)$ is called a mild solution of equations $(3)-(4)$ and is not necessarily a solution of equations $(3)-(4)$. 
Definition 1. A function $u(t)$ is a mild solution of equations $(3)-(4)$ on $[0, b)$ if $u \in C\left([0, b) ; X_{\alpha}\right), u(0)=u_{0}-g$, and $u(t)$ satisfies the integral equation $(5)$ on $[0, b)$.

Definition 2. A function $u(t)$ is a strong solution of equations (3) - (4) if $u \in$ $\left.C\left([0, b) ; X_{\alpha}\right)\right) \cap C^{1}([0, b) ; X), u(0)=u_{0}-g$, and $u(t)$ satisfies $(3)-(4)$ on $[0, b)$.

\section{Existence of Solutions}

We shall make the following assumptions on the operator $A$ and the nonlinear operators $f$ and $g$ :

(i) $-A$ is the infinitesimal generator of a bounded analytic semigroup of linear operator $T(t), t>0$, in $X$.

(ii) There exist real constants $M$ and $\delta$ such that $\|T(t)\| \leq M e^{\delta t}$ for $t>0$.

(iii) For $0 \leq \alpha<1$, the fractional power $A^{\alpha}$ satisfies $\left\|A^{\alpha} T^{\top}(t)\right\| \leq C_{\alpha} t^{-\alpha}$ for $t>0$ where $C_{\alpha}$ is a real constant.

We shall assume $X_{\alpha} \subseteq Y \subseteq X$ so that $T(t): X \rightarrow Y$ for all $t>0$ is a bounded linear operater and

(iv) $A^{\beta} T(t): X \rightarrow Y$ for $t>0$ and $\left\|A^{\beta} T(t)\right\| \in L^{1}(0, r)$ for $\beta \in[\alpha, \alpha+d]$ for some $d>0$ and every $r>0$.

(v) The function $f$ maps $X_{\alpha}$ into $X$, and satisfies:

there exists $G: Y \rightarrow X$ such that $\|G(u)-G(v)\| \leq K\|u-v\|$ where $K$ is a constant, $G: X_{\alpha} \rightarrow X_{\alpha}$ and for each $u(0) \in X_{\alpha}, f(u(0))=A^{\alpha} G(u(0))$

(vi) The function $g\left(t_{1}, \ldots, t_{p}, u\left(t_{1}\right), \ldots, u\left(t_{p}\right)\right)$ maps $J^{p} \times X_{\alpha}^{p}$ into $Y$ and satisfies:

there exist $h\left(t_{1}, \ldots, t_{p}, u\left(t_{1}\right), \ldots, u\left(t_{p}\right)\right): J^{p} \times X^{p} \rightarrow X$ and a constant $K_{1}>0$ such that

$$
\begin{aligned}
& \left\|h\left(t_{1}, \ldots, t_{p}, u\left(t_{1}\right), \ldots, u\left(t_{p}\right)\right)-h\left(t_{1}, \ldots, t_{p}, v\left(t_{1}\right), \ldots, v\left(t_{p}\right)\right)\right\| \leq K_{1}\|u-v\|, \\
& h: J^{p} \times X_{\alpha}^{p} \rightarrow X_{\alpha} \text { and } g=A^{\alpha} h .
\end{aligned}
$$

Theorem 1. If the assumptions $(i)$ to (vi) hold, then for each $u(0) \in Y$ there exists $a b>0$ and $a$ unique continuous function $u:[0, b) \rightarrow Y$ such that

$$
u(t)=T(t) u_{0}-T(t) A^{\alpha} h\left(t_{1}, \ldots, t_{p}, u\left(t_{1}\right), \ldots, u\left(t_{p}\right)\right)+\int_{0}^{t} A^{\alpha} T(t-s) G(u(s)) d s, \quad t \geq 0
$$

Proof. Define the set $S=\{u:[0, t] \rightarrow Y: u(t)$ and $g \in Y$ are continuous, $u(0)=$ $u_{0}-g$ and $\left.\|u(t)-u(0)\| \leq R\right\}$. Choose ' $b$ ' such that for

$$
\begin{array}{cc} 
& \|(T(t)-I)\|\left(\left\|u_{0}\right\|+\left\|g\left(t_{1}, \ldots, t_{p}, u\left(t_{1}\right), \ldots, u\left(t_{p}\right)\right)\right\|\right) \\
+C b^{(1-\alpha)} /(1-\alpha)\{\|G(u(0))\|+K R\} \leq R \\
\text { and } \quad\left\{\left\|A^{\alpha} T(t)\right\| K_{1}+K b^{(1-\alpha)} /(1-\alpha)\right\}<1
\end{array}
$$


Moreover, define the mapping $P$ on $S$ by

$$
(P u)(t)=T(t) u_{0}-T(t) g\left(t_{1}, \ldots, t_{p}, u\left(t_{1}\right), \ldots, u\left(t_{p}\right)\right)+\int_{0}^{t} A^{\alpha} T(t-s) G(u(s)) d s, \quad t \geq 0
$$

First note that for $u \in S, P$ is well defined since

$$
\begin{aligned}
\int_{0}^{t}\left\|A^{\alpha} T(t-s) G(u(s))\right\| d s & \leq\left\{\int_{0}^{t} A^{\alpha} T(t-s) \| d s\right\} \cdot\{\|G(u(0))+G(u(t))-G(u(0))\|\} \\
& \leq\left\{\int_{0}^{t}\left\|A^{\alpha} T(t-s)\right\| d s\right\} \cdot\{\|G(u(0))\|+K R\}
\end{aligned}
$$

For $u \in S$, we have

$$
\begin{aligned}
\|(P u)(t)-u(0)\|= & \| T(t) u_{0}-T(t) g\left(t_{1}, \ldots, t_{p}, u\left(t_{1}\right), \ldots, u\left(t_{p}\right)\right) \\
& +\int_{0}^{t} A^{\alpha} T(t-s) G(u(s)) d s-\left(u_{0}-g\right) \| \\
\leq & \|(T(t)-I)\|\left(\left\|u_{0}\right\|+\left\|g\left(t_{1}, \ldots, t_{p}, u\left(t_{1}\right), \ldots, u\left(t_{p}\right)\right)\right\|\right) \\
& +C b^{(1-\alpha)} /(1-\alpha)\{\|G(u(0))\|+K R\} \\
\leq & R
\end{aligned}
$$

This implies that $P(S) \subseteq S$. Therefore, $P$ maps $S$ into itself. Let $u, v \in S$, then we have

$$
\begin{aligned}
& \|(P u)(t)-(P v)(t)\| \\
\leq & \left\|T(t) A^{\alpha}\left(h\left(t_{1}, \ldots, t_{p}, u\left(t_{1}\right), \ldots, u\left(t_{p}\right)\right)-h\left(t_{1}, \ldots, t_{p}, v\left(t_{1}\right), \ldots, v\left(t_{p}\right)\right)\right)\right\| \\
& +\left\|\int_{0}^{t} A^{\alpha} T(t-s)[G(u(s))-G(v(s))] d s\right\| \\
\leq & \left\|A^{\alpha} T(t)\right\| K_{1}\|u-v\|+\left\{K \int_{0}^{t}\left\|A^{\alpha} T(s)\right\| d s\right\}\|u-v\| \\
\leq & \left\{\left\|A^{\alpha} T(t)\right\| K_{1}+K \int_{0}^{t}\left\|A^{\alpha} T(s) d s\right\|\right\}\|u-v\| \\
\leq & \left.\left\{\left\|A^{\alpha} T(t)\right\| K_{1}+K b^{1-\alpha} / 1-\alpha\right)\right\}\|u-v\|
\end{aligned}
$$

By the contraction mapping theorem $P$ has a unique fixed point $u \in S$.

Lemma $\mathbb{1}$. Let assumptions $(i)-(v i)$ be satisfied, then all $\theta>0$ and $t, t+h \in[\theta, a]$ there exist $\sigma$ such that $\|u(t+h)-u(t)\| \leq C(\theta) h^{\sigma}, 0<\sigma<1$.

Proof. Now

$$
\begin{aligned}
\|u(t+h)-u(t)\| \leq & \left.\|(T(h)-I) T(t) u_{0}\right)\|+\|(T(h)-I) A^{\alpha} T(t) h\left(t_{1}, \ldots, t_{p}, u\left(t_{1}\right), \ldots, u\left(t_{p}\right)\right) \| \\
& +\left\|\int_{0}^{t} A^{\alpha}\{(T(h)-I) T(t-s)\} G(u(s)) d s\right\|
\end{aligned}
$$




$$
\begin{aligned}
& +\left\|\int_{t}^{t+h} A^{\alpha} T(t+h-s) G(u(s)) d s\right\| \\
\leq & \left\|A^{\alpha} T(t)(T(h)-I) A^{-\alpha} u_{0}\right\| \\
& +\left\|A^{\alpha} T(t)(T(h)-I) h\left(t_{1}, \ldots, t_{p}, u\left(t_{1}\right), \ldots, u\left(t_{p}\right)\right)\right\| \\
& +\left\|(T(h)-I) \int_{0}^{t} A^{\alpha} T(t-s) G(u(s)) d s\right\| \\
& +\left\|\int_{t}^{t+h} A^{\alpha} T(t+h-s) G(u(s)) d s\right\| \\
\leq & C \theta^{-\tau}\left\|(T(h)-I) A^{-\alpha} u_{0}\right\| \\
& \left.+C \theta^{-\tau} \| T(h)-I\right) A^{-\alpha} A^{\alpha} T(t) h\left(t_{1}, \ldots, t_{p}, u\left(t_{1}\right), \ldots, u\left(t_{p}\right)\right) \| \\
& +\left\|\int_{0}^{t} A^{\alpha+\epsilon} T(t-s)(T(h)-I) A^{-\epsilon} G(u(s)) d s\right\| \\
& +\int_{t}^{t+h} A^{\alpha} T(t+h-s) d s \cdot\{\|G(u(0))\|+K R\} \\
\leq & C \theta^{-\tau}\left\|u_{0}\right\| h^{\alpha}+C \theta^{-\tau}\left\|A^{\alpha} T(t) h\left(t_{1}, \ldots, t_{p}, u\left(t_{1}\right), \ldots, u\left(t_{p}\right)\right)\right\| h^{\alpha} \\
& +\left[C(\|G(u(0))\|+K R) \int_{0}^{t}\left\|A^{\alpha+\epsilon} T(s)\right\| d s\right\} h^{\epsilon}+C\{\|G(u(0))\|+K R\} h^{1-\tau}
\end{aligned}
$$

Taking $\sigma=\min \{\alpha, 1-\tau, \varepsilon\}$, where $0<\varepsilon<\sigma$, hence the Lemma.

This establishes that a solution of equation (6) is locally Holder continuous on $(0, b]$. If the solution $u(t)$ of equation (6) is in $X_{\alpha}$ and if it is also Holder continuous in the $X_{\alpha}$ norm we can show that $u(t)$ is a solution of $(3)-(4)$ if $f$ and $g$ are locally Lipschitz continuous from $X_{\alpha}$ into $X$ and $J^{p} \times X_{\alpha}^{p}$ into $Y$ respectively.

Lemma 2. Let assumptions of Lemma 1 be hold. Then the solution $u(t)$ of equation (6) is in $X_{1-\alpha}$ for $t \in(0, b]$.

Proof. Let $\mu>0$, then the solution $u(t)$ of equation (6) satisfies

$$
\begin{aligned}
u(t)= & T(t-\mu) u_{0}-T(t-\mu) A^{\alpha} h\left(t_{1}, \ldots, t_{p}, u\left(t_{1}\right), \ldots, u\left(t_{p}\right)\right) \\
& +\int_{\mu}^{t} A^{\alpha} T(t-s) G(u(s)) d s
\end{aligned}
$$

and

$$
\begin{aligned}
u(t)= & T(t-\mu) u_{0}-T(t-\mu) A^{\alpha} h\left(t_{1}, \ldots, t_{p}, u\left(t_{1}\right), \ldots, u\left(t_{p}\right)\right) \\
& +\int_{\mu}^{t} A^{\alpha} T(t-s) G(u(t)) d s+\int_{\mu}^{t} A^{\alpha} T(t-s)[G(u(s))-G(u(t))] d s
\end{aligned}
$$

Since $T(t-\mu) u_{0}, T(t-\mu) A^{\alpha} h\left(t_{1}, \ldots, t_{p}, u\left(t_{1}\right), \ldots, u\left(t_{p}\right)\right) \in D(A)$ for all $t>\mu$ and $\int_{\mu}^{t} A^{\alpha} T(t-s) G(u(t)) d s=A^{\alpha-1}[G(u(t))-T(t-\mu) G(u(t))]$ for all $t \geq \mu$. We have only 
to show that

$$
\int_{\mu}^{t} A^{\alpha} T(t-s)[G(u(s))-G(u((t))] d s
$$

is in $X_{1-\alpha}$. Note that

$$
\begin{aligned}
\left\|A^{1-\alpha} \int_{\mu}^{t} A^{\alpha} T(t-s)[G(u(s))-G(u(t))] d s\right\| & =\left\|\int_{\mu}^{t} A T(t-s)[G(u(s))-u(t)] d s\right\| \\
& \leq C K \int_{\mu}^{t}(t-s)^{-1}(t-s)^{\sigma} d s \\
& =C K(t-\mu)^{\sigma} / \sigma .
\end{aligned}
$$

By Lemma 1, the last inequality is true.

Lemma 3. Let the assumptions of the previous Lemma holds and that $X_{1-\alpha} \subseteq X_{\alpha}$, the imbedding being continuous, then the solution $u(t)$ of equation (6) is a mild solution of equations (3) - (4) and is locally Holder continuous into $X_{\alpha}$.

Proof. From Lemma 2 and assuption $X_{1-\alpha} \subseteq X_{\alpha}$ implies that $u(t) \in X_{\alpha}$ for all $t>0$. Thus for $\mu>0$ and $t>\mu / 2$ we have

$$
\begin{aligned}
u(t)= & T(t-\mu / 2) u_{0}-T(t-\mu / 2) g\left(t_{1}, \ldots, t_{p}, u\left(t_{1}\right), \ldots, u\left(t_{p}\right)\right) \\
& +\int_{\mu / 2}^{t} T(t-s) f(u(s)) d s
\end{aligned}
$$

Since $u(t)$ is continuous into $X_{1-\alpha}$ and $X_{1-\alpha}$ is continuously imbedded into $X_{\alpha}, u(t)$ is continuous into $X_{\alpha}$. Now we show that $u(t)$ is locally Holder continuous into $X_{\alpha}$. Let $\mu>0$ and $t+h, t \in[\mu, b]$, then

$$
\begin{aligned}
\|u(t+h)-u(t)\| \leq & \left\|A^{\alpha}(T(h)-I) T(t-\mu / 2) u_{0}\right\| \\
& +\left\|A^{\alpha}(T(h)-I) T(t-\mu / 2) g\left(t_{1}, \ldots, t_{p}, u\left(t_{1}\right), \ldots, u\left(t_{p}\right)\right)\right\| \\
& +\left\|\int_{\mu / 2}^{t} A^{\alpha} T(t-s)(T(h)-I) f(u(s)) d s\right\| \\
& +\left\|\int_{t}^{t+h} A^{\alpha} T(t+h-s) f(u(s)) d s\right\| \\
\leq & C\left\|u_{0}\right\| h^{\alpha}+C\left\|g\left(t_{1}, \ldots, t_{p}, u\left(t_{1}\right), \ldots, u\left(t_{p}\right)\right)\right\| h^{\alpha} \\
& +\left\{C \int_{\mu / 2}^{t}\left\|A^{\alpha+\varepsilon} T(t-s)\right\| d s\right\} \sup \|f(u(s))\| h^{\varepsilon} \\
& +C /(1-\alpha) \sup \|f(u(t))\| h^{1-\alpha}
\end{aligned}
$$

where $\varepsilon$ is chosen so that $\alpha+\varepsilon<1$. Thus there exist a $C>0$ and a $0<\theta<1$ such that $\|u(t+h)-u(t)\| \leq C h^{\theta}$ for $t, t+h, \in[\mu, b]$. 
Theorem 2. If the conditions $(i)$ - (vi) and the assumptions of Lemma 3 are satisfied, and $f$ and $g$ are locally Lipshitz from $X_{\alpha}$ into $X$ and $J^{p} \times X_{\alpha}^{p}$ into $Y$ respectively, then any solution of equation (6) is also a strong solution of equations (3) - (4).

Proof. Since $f$ and $g$ are locally Lipshitz and $u(t)$ is locally Holder continuous into $X_{\alpha}$, the fuctions $f$ and $g$ are locally Holder continuous on $[\mu, b]$ for any $\mu>0$. Thus the theory of analytic semigroups of linear operators [12] gives the desired result.

Theorem 3. Let the assumptions of Lemma 1 be satisfied, then $u(t)$ may be extended to a maximum interval of existence $I=[0, c)$, where $c=\max b$. If $b<\infty$ then

$$
\begin{aligned}
& \lim _{t \rightarrow c} \int_{0}^{t}\left\|A^{\alpha} T(t-s)\right\|\|G(u(s))\| d s=\infty \\
& \lim _{t \rightarrow c}\|u(t)\|=\infty
\end{aligned}
$$

Proof. Suppose $c<\infty$. For every $t \in I, u(t)$ satisfies the integral equation (6). Claim $\lim _{t \rightarrow c} \sup \|u(t)\| \leq C$ for all $t \in I$ and some $C>0$. This gives

$$
\begin{aligned}
\|u(t)\| \leq & \left\|T(t) \mu_{0}\right\|+\left\|T(t) A^{\alpha} h\left(t_{1}, \ldots, t_{p}, u\left(t_{1}\right), \ldots, u\left(t_{p}\right)\right)\right\| \\
& +\left\{\int_{0}^{c}\left\|A^{\alpha} T(t-s)\right\| d s\right\} \cdot\left\{\sup _{t \in I}\|G(u(t))\|\right\}
\end{aligned}
$$

For $0<\tau<t<c$, we have

$$
\begin{aligned}
& \|u(t)-u(\tau)\| \\
\leq & \left\|A^{\alpha} T(\tau)[T(t-\tau)-I] A^{-\alpha} u_{0}\right\| \\
& +\left\|A^{\alpha} T(\tau)[T(t-r)-I] A^{-\alpha} A^{\alpha} h\left(t_{1}, \ldots, t_{p}, u\left(t_{1}\right), \ldots, u\left(t_{p}\right)\right)\right\| \\
& +\left\|\int_{0}^{\tau} A^{\alpha+\epsilon} T(\tau-s)[T(t-\tau)-I] A^{-\epsilon} G(u(s))\right\| d s+\left\|\int_{\tau}^{t} A^{\alpha} T(t-s) G(u(s))\right\| d s \\
\leq & C \tau^{-\varepsilon}\left\|u_{0}\right\|(t-\tau)^{\alpha}+C \tau^{-\varepsilon}\left\|A^{\alpha} h\left(t_{1}, \ldots, t_{p}, u\left(t_{1}\right), \ldots, u\left(t_{p}\right)\right)\right\|(t-\tau)^{\alpha} \\
& +C(t-\tau)^{\varepsilon}\left\{\int_{0}^{\tau}\left\|A^{\alpha+\varepsilon} T(s) d s\right\|\right\} \cdot\left\{\sup _{t \in I}\|G(u(t))\|\right\} \\
& +\left\{\int_{\tau}^{t}\left\|A^{\alpha} T(s) d s\right\|\right\} \cdot\left\{\sup _{t \in I}\|G(u(t))\|\right.
\end{aligned}
$$

Thus we have $\|u(t)-u(\tau)\|=0$ as $t, \tau \rightarrow c$, contradicting the maximality of $c$.

If $\lim _{t \rightarrow c}\|u(t)\| \neq \infty$ then there exist numbers $r>0$ and $d>0$ with $d$ arbitrarily large and sequences $\tau_{n} \rightarrow c, t_{n} \rightarrow c$ as $n \rightarrow \infty$ and such that $\tau_{n}<t_{n}<c .\left\|u\left(\tau_{n}\right)\right\|=r$, $\left\|u\left(t_{n}\right)\right\|=r+d$ and $\|u(t)\| \leq r+d$ for $t \in\left[\tau_{n}, t_{n}\right]$. We have

$$
\begin{aligned}
\left\|u\left(t_{n}\right)-u\left(\tau_{n}\right)\right\| \leq & \left\|\left[T\left(t_{n}-\tau_{n}\right)-I\right] u\left(\tau_{n}\right)\right\| \\
& +\left\|A^{\alpha}\left[T\left(t_{n}-\tau_{n}\right)-I\right] h\left(t_{1}, \ldots, t_{p}, u\left(t_{1}\right), \ldots, u\left(t_{p}\right)\right)\right\| \\
& +\left\|\int_{\tau_{n}}^{t_{n}} A^{\alpha} T\left(t_{n}-s\right) G(u(s))\right\|
\end{aligned}
$$


The right hand side of (10) approaches zero as $\tau_{n}$ and $t_{n}$ approach $c$ while the left hand side of (10) is bounded below by $d>0$. This contradiction gives the result and ( 8 ) follows from (9).

\section{References}

[1] K. Balachandran and S. Ilamaran, Existence and uniqueness of mild and strong solutions of a semilinear evolution equation with nonlocal conditions, Indian J. Pure Appl. Math. 25 (1994), 411-418.

[2] K. Balchandran and M. Chandrasekaran, Existence of solution of a delay differential equation with nonlocal conditions, Indian J. Pure Appl. Math. 27 (1996), 443-449.

[3] L. Byszewski, Existence and uniqueness of solutions of nonlocal problems for hyperbolic equation $u_{x t}=F\left(x, t, u, u_{x}\right)$, J. Math. Stoch. Anal., 3 (1990), 163-168.

[4] L. Byszewski, Theorem about existence and uniqueness of continuous solution of nonlocal problem for nonlinear hyperbolic equation, Appl. Anal. 40 (1991), 173-180.

[5] L. Byszewski, Theorems about the existence and uniqueness of solutions of a semilinear evolution nonlocal Cauchy problem, J. Math. Anal. Appl. 162 (1991), 494-506.

[6] L. Byszewski, Existence of approximate solution to abstract nonlocal Cauchy problem, J. Appl. Math. Stoch. Anal. 5 (1992), 363-374.

[7] L. Byszewski, Uniquness criterion for solution of abstract nonlocal Cauchy problem, J. Appl. Math. Stoch. Anal. 6 (1993), 49-54.

[8] A. Friedman, Partial Differential Equations, Renehart and Winston, New York, 1969.

[9] D. Henry, Geometric Theory of Semilinear Parabolic Equation, Springer-Vertag, New York, 1981.

[10] D. Jackson, Existence and uniqueness of solutions to semilinear nonlocal parabolic equations, J. Math. Anal. Appl. 172 (1993), 256-265.

[11] Y. Lin and J. H. Liu, Semilinear integrodifferential equations with nonlocal Cauchy problem, Nonlinear Analysis, Theory, Method and Appl. 26 (1996), 1023-1033.

[12] A. Pazy, Semigroups of Linear Operators and Applications to Partial Differential Equations, Springer-Verlag, New York, 1983.

[13]. S. M. Rankin III, Semilinear evolution equations in Banach spaces with application to parabolic partial differential equations, Trans. Amer. Math. Soc. 336 (1993), 523-535.

Department of Mathematics, Bharathiar University, Coimbatore 641 046, India. 